\title{
DỨBin
}

Technological University Dublin

ARROW@TU Dublin

2015-02-05

\section{An Analytical Representation of Flexible Resource Allocation in Hospitals}

\author{
Michael Thorwarth \\ Technological University Dublin \\ Wael Rashwan \\ Technological University Dublin, wael.rashwan@tudublin.ie \\ Amr Arisha \\ Technological University Dublin, amr.arisha@tudublin.ie
}

Follow this and additional works at: https://arrow.tudublin.ie/buschmarart

Part of the Business Administration, Management, and Operations Commons, Management Sciences and Quantitative Methods Commons, and the Operations and Supply Chain Management Commons

\section{Recommended Citation}

Thorwarth, M., Rashwan, W., \& Arisha, A. (2015), An analytical representation of flexible resource allocation in hospitals, Flexible Services and Manufacturing Journal, Vol. 26, Issues 93, pp 1989 - 2014, Springer US, DOI: 10.1007/s10696-015-9216-4.

This Article is brought to you for free and open access by the School of Marketing at ARROW@TU Dublin. It has been accepted for inclusion in Articles by an authorized administrator of ARROW@TU Dublin. For more information, please contact arrow.admin@tudublin.ie, aisling.coyne@tudublin.ie,gerard.connolly@tudublin.ie.

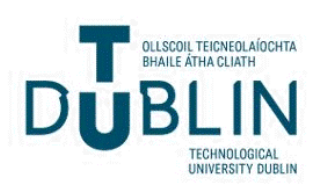


Noname manuscript No.

(will be inserted by the editor)

\title{
An Analytical Representation of Flexible Resource Allocation in Hospitals
}

\author{
Michael Thorwarth • Wael Rashwan • \\ Amr Arisha
}

Received: date / Accepted: date

\begin{abstract}
Non-linear processes within complex systems are difficult to predict. Using Discrete Event Simulation (DES) models can be helpful for presenting the uncertainty level embedded within these processes. However, the interpretations of the resulting outcomes can be challenging to analyze. This is evident when experimenting with simulation models, and therefore requires the adaptation of existing models. Introducing cyclic entities such as interconnected resources to the simulation model adds a new level of complexity to the
\end{abstract}

Michael Thorwarth

Dublin Institute of Technology

College of Business

Aungier Street

Dublin, 2

Ireland

Tel.: +49-160-95809487

E-mail: Michael.Thorwarth@gmx.net

Present address: of Michael Thorwarth

Sudetenstr. 7

D-49076 Osnabrueck

Germany

Amr Arisha

Dublin Institute of Technology

College of Business

Aungier Street

Dublin, 2

Ireland

Wael Rashwan

Dublin Institute of Technology

College of Business

Aungier Street

Dublin, 2

Ireland 
model. In practical cases, decision makers often demand a model that enables them to make quick decisions with less complexity involved. However, these individuals still want to be assured of a high degree of accuracy in the results. This article demonstrates how an analytical representation of complex DES models can be developed in order to facilitate prompt yet effective solutions for decision makers. This analytical representation provides a brief overview of the simulation results by using the staff scheduling and nurses' utilization approaches. Results of the effective use of DES model and managers feedback are encouraging.

Keywords Analytical Representation · Process Management · Discrete Event Simulation · Queuing Theory

\section{Introduction}

Healthcare managers are close to accepting and appreciate the value of the application of Discrete Event Simulation (DES) in healthcare planning and management activities. It provides them with a reliable and flexible tool which allows them to forecast demand for services, allocate resources, and analyze and optimize their system parameter settings. Current and past research have also revealed the complexity among interlinked resources and their function in a multiple flows environment [18]. The technique that facilitates the combining of different process flows within one model is known as Multiple Participant Pathway Modeling (MPPM), where both active and passive pathways are described by the nature of the resources applied to them. Those resources which drive system processes are labeled as 'active contributors', and those mainly concerned with receiving the services are described as 'passive practitioner'. Resources remain passive when no active pathway is involved. Employing both active and passive pathways within the same simulation model add a significant degree of complexity to the model. Hence practitioners demand a simpler approach with the aim of deriving some general rules of the thumb to determine if some scenarios appear frequently in their real-world systems. For this reason, this article illustrates how a complex simulation model can be transformed into an analytical representation which can then be applied as a formula in spreadsheet applications. While such analytical representations are subject to certain boundaries and constraints, these are manageable for operational decision makers who need to be able to make quick but correct choices.

\section{Background}

The underlying simulation modeling allows the combining of passive patient pathways with the active pathways of staff workflows, and is examined with the use of a theoretical evaluation to highlight the applicability of the concept 
within the constraints of queuing theory. This article also aims to identify particular regularities and limitations in the MPPM concept as described in Thorwarth [18] by investigating Flexible Resource Allocation (FRA). Research on the implementation of simulation applications tend to focus more on manufacturing and production systems. However, the dynamics of service applications receive less attention. While the flexible workload concept is often described as multitasking, it should not be mistaken with the normal use of the term which means more than one task to be undertaken at the same time in parallel. In this context, flexible workload describes a worker dealing with several different tasks sequentially, in a flexible manner, within a given time frame. This phenomenon can be observed in hospital emergency departments (ED), where medical staff members deal with various patients, probably involving various treatments, within the same time frame. In general, flexible workload environments appear complex and highly dynamic to the observer. This article aims (1) to understand the complexity of the flexible workload within the process chain in ED, and (2) to develop an analytical representation that can help managers to allocate staff to meet patient demand. The analytical representation illustrates the flexible utilization of staff members depending on hourly patient arrival numbers. Furthermore, it allows for the adjustment of the number of processes within the process chain, which makes it attractive for healthcare managers who face changing process settings within their departments. While it cannot substitute for a thorough simulation analysis of the department's activities, an analytical representation is an abstract approach that managers can use as a quick operational guide to help with resource and staff allocation decisions.

\subsection{Literature Review}

Healthcare systems are human-based systems that involve multiple stakeholders interacting with each other in complex ways. Due to the stochastic nature of healthcare systems and the complex dynamics and interactions of their inputs, activities and outputs, healthcare providers need tools that enable them to understand this complexity and in this way enhance their system performance. DES is a useful tool for assisting healthcare managers in making their decisions [10]. A simulation can map patient flows, routing layouts, staffing, procedures, and equipment allocations so that optimal departmental control strategies may be developed. DES has been proven to be an effective tool for process modeling and improvement of healthcare applications [6]. Healthcare managers can use DES models to assess the current performance of systems, predict the impact of operational changes, and examine the trade-offs among system variables [20]. DES was also used in many applications in the healthcare context, for example: 
- to map patient flows, and identify bottlenecks in the provision of acute care beds and rehabilitation services in the provision of care for elderly patients in Ireland [15];

- potential service improvement through possible reorganization of existing resources [17];

- resource planning and assessing the risks of staff shortages [9];

- planning the geographical locations of new healthcare facilities while taking into account population demographics and the locations of (potential) patients who will need to use the services [5].

In EDs, DES is utilized to address problems such as staff scheduling under uncertainty, and planning new EDs [1].

Research has reported some drawbacks in the use of simulation modeling in general. This includes that it can be time consuming due to multiple simulation runs for statistically valid results $[2,14]$. Moreover, expertise is required to develop and run simulation models which discourages healthcare managers from using it $[12,13]$.

One common objective of simulation models is to help users to gain a better understanding of the relationships between the input and output variables of real-world systems. With a simple, analytical approach such as an auxiliary tool the simulation model has advantages in providing a better understanding of complex systems and guiding ad hoc decision making, as well as providing a framework for testing alternatives and optimizations [3]. Understanding, however, is a broad term and describes an abstract learning effect by the applicant of the simulation model. In order to be more precise about the learning process and the level of understanding, this articles provides an analytical representation which describes the system under investigation by using DES. The authors of this paper have, to the best of their knowledge, not retrieved similar descriptions of the system.

Common objective of simulation models is to help users to gain a better understanding of the relations between the input and output variables of real systems. Analytical representations of simulation models can be effective tool in explaining and interpreting complex detailed systems. Although simulation models are considered as simplifications of the real world, gaining understanding of how inputs are related to outputs can still be very difficult, so providing a simple, analytical model as an auxiliary tool the simulation model has advantages in delivering better understandings of complex systems and guiding ad hoc decisions, and also providing a framework for testing alternatives and optimizations [3]. 


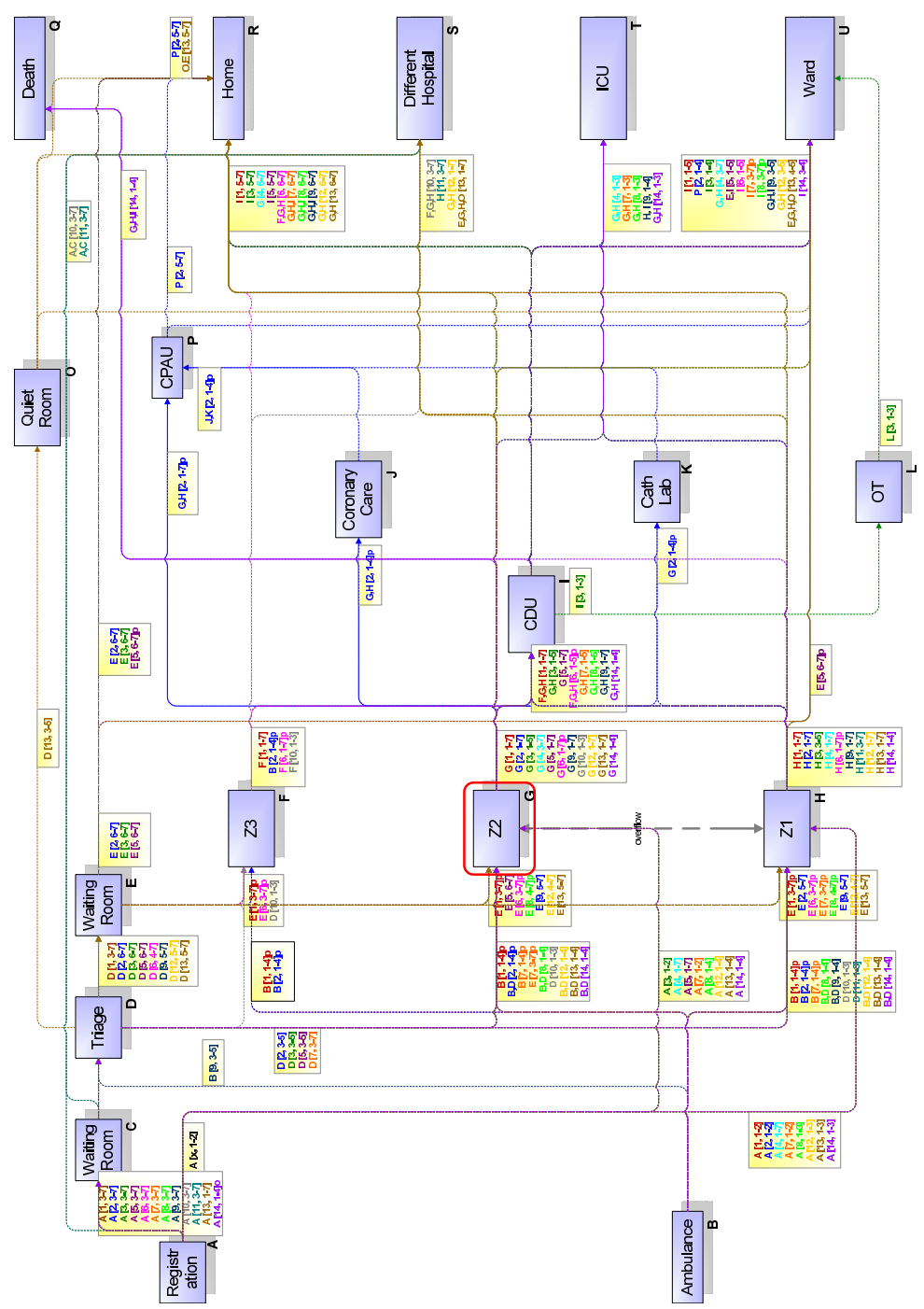

Fig. 1 Flowchart illustrating the potential paths of patients through the ED.

\subsection{Description of the Underlying Simulation Model}

A detailed simulation modeling for an ED was developed with the objective of analyzing resources and patient flow. The ED is located in a university hospital that serves approximately 50,000 patients annually. EDs in Ireland provide a wide range of care services and consequently face high levels of demand which result in emergency patients having to stay in EDs for significant periods of time [7]. Accordingly, ED managers struggle to find solutions for bottlenecks and to optimize their patient flows. 
Figure 1 depicts a flowchart to illustrate the complexity of the ED processes. The flow chart displays units (blue boxes) through which patients travel. Beside the units are yellow labels that indicate the patient class and, accordingly, its severity level (indicated in brackets). The letter shows the subsequent unit (A to U). The flowchart therefore indicates the ED layout and treatment zones. The ED has three central units: the chest pain unit (Z1), the general unit (Z2), and the minor injuries unit (Z3). The minor injuries unit Z3 (in red and labeled $\mathrm{F}$ in the figure 1 ) is the ED's most frequently visited unit, and primarily treats trauma patients as well as those with general complaints of minor severity. A patient entering this unit follows a linearly assigned process chain of treatments and activities. The analytical representation which is developed here focuses on the process chain of trauma patients, but a similar representation could also be applied to the other two central units (Z1 and Z2). The aim of this article is to develop an analytical representation that examines and describes the mechanisms of flexible resource allocation in the ED.

Most of the staff within the ED are dedicated to its tasks and units. However, some of the staff, especially nurses, are qualified for more than just one unit and therefore their allocation is flexible. In addition, there are nurses that can come in on call whenever the demand is high. Flexible allocation in combination with nurses that can come in on demand is referred as staff pooling and proves to be a necessary tool to meet peaks in patient demands.

The ED can be characterized as a set of several integrated and connected processes. The focus of this study is on the staff who alternate between various tasks in this highly dynamic environment. The nurses' work can be best described as a spontaneous allocation of their work to wherever it is needed most. They complain about the high workload and stress level caused by this practice which they refer to as 'multitasking'. Some nurses are qualified to work on all tasks, so they can be used to back up staff in other units where the workload is higher than planned or expected. This poolin is one of the reasons why this analytical representation is a helpful tool for the ED manager: $\mathrm{s} /$ he can use it to decide quickly whether a nurse has to be taken out of the pool or not. Nurses are the ED's only pooled staff resource addressed in this article because they are the primary focus of the study.

The simulation model is derived from descriptions and observation. This alternating of staff between different tasks within the process chain can be modeled using DES because of the flexibility and robustness of the approach. However, an algebraic expression needs to be identified which can represent the mechanism of flexible work allocation. Deriving this algebraic expression is thus the first step in the optimization process which aims to achieve the optimum balance between staffing levels and service quality.

The following section demonstrates the comprehensive application of a simulation that analyzes the complex dynamic environment of the uncertain sys- 
tem being investigated. Moreover, it describes the relationship between various entities within the system. This analytical model provides a better understanding of the system, supports the optimization process and provides healthcare decision makers with a formula that can be used to calculate staff utilization and indicate the threshold numbers of staff required to deliver quality service.

\subsection{Flexible Workload Model}

To derive an analytical representation of a process chain including the required FRA, staff utilization is used as the key indicator for demonstrating the impact of uncertain patient arrivals, resources (mainly beds, cubicles and instruments) and staff availability within the process chain. The description of the utilization $\rho$ depends on the patients' inter-arrival time $T$ and the number of available staff $n$ is based on the model (Figure 2). This process chain is a one dimensional representation of $m_{i}$ sequential process elements that are completed by $n$ personnel, who are allocated from the staff pool. Personnel circulate throughout the process chain and are allocated to the process task where their presence is required - similar to a pull system. The parameter $M$ is the number of tasks within the process chain which can only be finalized by an appropriately trained staff member who can complete the process and release the patient to the next process stage: so patient must wait until a nurse with the relevant seniority and experience is available. A higher logic level (which is also built into the simulation model) assigns greatest priority to the patients who are furthest along in the process chain in order to counter unwanted blockages. However, queues between the processes act as buffers which can also be investigated.

The following example explains the ED's flexible workload allocation, the patient arrival rate, is set to $\lambda$. Patients register and wait in the waiting room while the first treatment process is occupied. Once it is free the patient enters the treatment chain at process $m_{1}$, which contains $M$ tasks. In the example shown in Table $1, M=6$, and the assigned nurse carries out the following tasks sequentially: triage; placing patient in a cubicle; taking samples as requested by the examiner; preparation for diagnosis; caring for patients' needs; and making arrangements for onward referral. In this context, the examiner is resource bound to this specific process element.

\section{Design of the Analytical Representation}

To design a effective analytical representation, the fundamental principles and mechanisms of the developed simulation model have to be analyzed. A detailed description of the full simulation model can be found in Thorwarth [18].The 


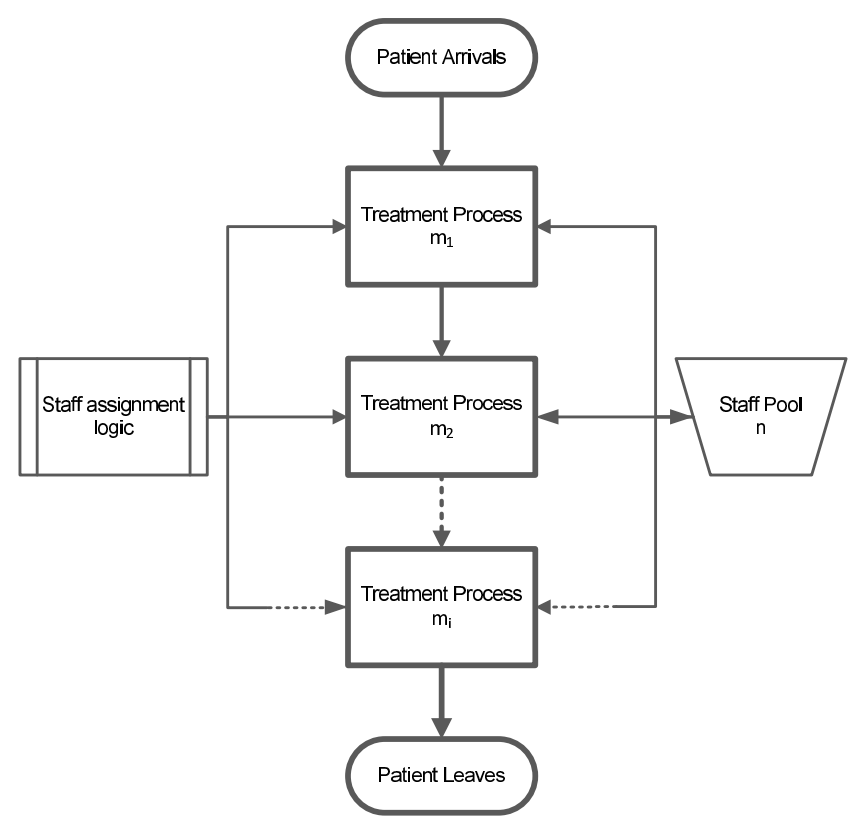

Fig. 2 Simplified multitasking model illustrating flexible resource allocation.

Table 1 Example of nursing tasks in a flexible workload environment.

\begin{tabular}{cl}
\hline $\begin{array}{c}\text { Task number } \\
i\end{array}$ & $\begin{array}{l}\text { Description of the task } m_{i} \\
\text { for nurse } j\end{array}$ \\
\hline 1 & Triage patient \\
2 & Place patient in cubicle / bed \\
3 & Take samples (blood, tissue, etc. as requested) \\
4 & Arrange diagnosis (x-ray, ultra sound, ct, etc.) \\
5 & Pay attention to patient needs \\
6 & Make arrangements for referral \\
\hline
\end{tabular}

focus of this study is on describing the derivation of the analytical representation from the simulation model.

\subsection{Simulation Results}

The analytical representation is derived from various repetitive simulation runs (Figure 3). Poisson distribution is used for patient arrivals times (taken from the hospital records) in the simulation model. A warm-up period to balance the queues of the simulation model has been implemented for the overall measurements. The process service times are assumed equal and normally distributed. 


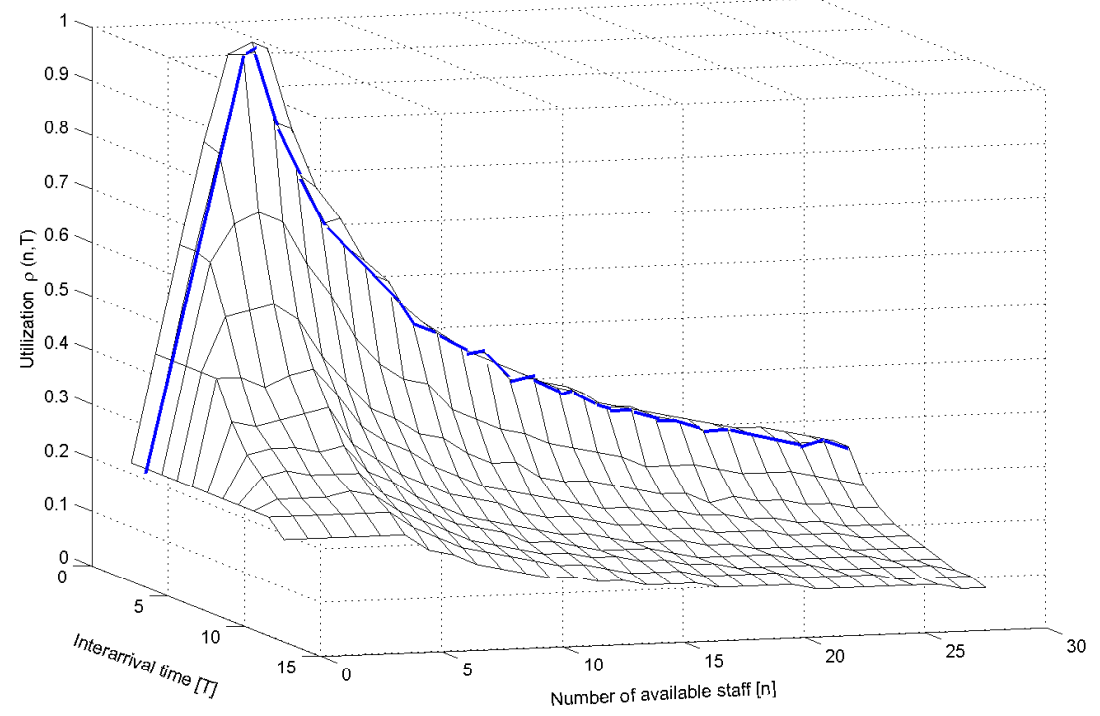

Fig. 3 The graph displays the resulting utilization $\rho(n, T)$ of a flexible workload environment depending on patient inter-arrival time $T$ and the number of the available staff $n$.

The overall utilization of each process chain $\rho(n, T)$ element depends on the available number of staff $n$ and the utilization of the element itself $\rho_{s}(n)$ where the patient is treated by the staff member, here referred as utilization activity $\rho_{a}(n, T)$.

\section{- INSERT FORMULA HERE}

The patients' inter-arrival times $T$ is the reciprocal of the arrival rate $\lambda$ and assumed to be stationary Poisson distributed in order to analyze and understand the core system behavior of the simulated ED model. In practice, the arrival rates are variant distributed processes, but in order to provide a tool for adjusting staffing levels in the ED, the focus is here is on the system behavior itself.

Figure 3 indicates several trends within the results plane, such as linear areas, a plateau and a hyperbola area, which reveal that several regularities are involved within the simulation model. These regularities can be used to derive an analytical representation. The overall utilization of the process chain is subdivided into its components (utilization of medical staff and activities). 


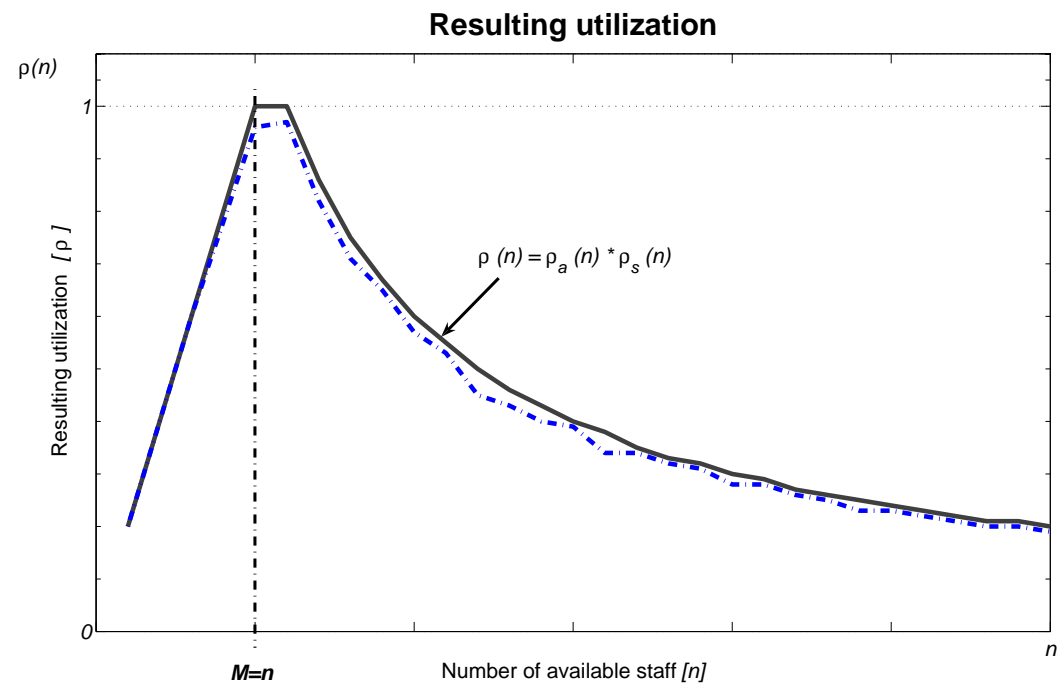

Fig. 4 The simulation result (dotted line) and the estimated calculated model (solid line) for the overall utilisation $\rho(n)$ for a specific arrival timeslot $t$ in dependence of the available staff $n$.

\subsection{Analytical Model}

DES is a practical platform to facilitate the illustration of processes in light of queuing theory. Law and Kelton [11] provide a comprehensive description of a DES application for queuing theory where the models investigated are usually described as being composed of tandem queues. The output of one stage affects the input of the next stage [4]. In the ED model studied in this article, the influences of the available staff $n$ and the number of tasks $M$ are investigated in relationship to patient inter-arrival times. In order to derive a function that can describe this flexible workload environment, the components of each result that are observable in the simulation results plane were investigated and interpreted separately, with the main focus on the number of staff available at a certain arrival time slot $t$ (Figure 4 ). For that reason figure 4 is cut along the time axis at a specific time which is referred to here as the time slot.

Simulation results for a specific inter-arrival time slot $t$ are shown by the blue dash-dotted line, and although statistical randomness causes some deviations, an overall trend can be clearly observed. In order to describe the trend, the components of the overall utilization should be examined. The overall utilization $\rho(n)$ is a combination of the utilization of staff $\rho_{n}(n)$ and the utilization of tasks expressed as activities $\rho_{a}(n)$, and is thus a combined process which depends on the staff member participating in the activity. 


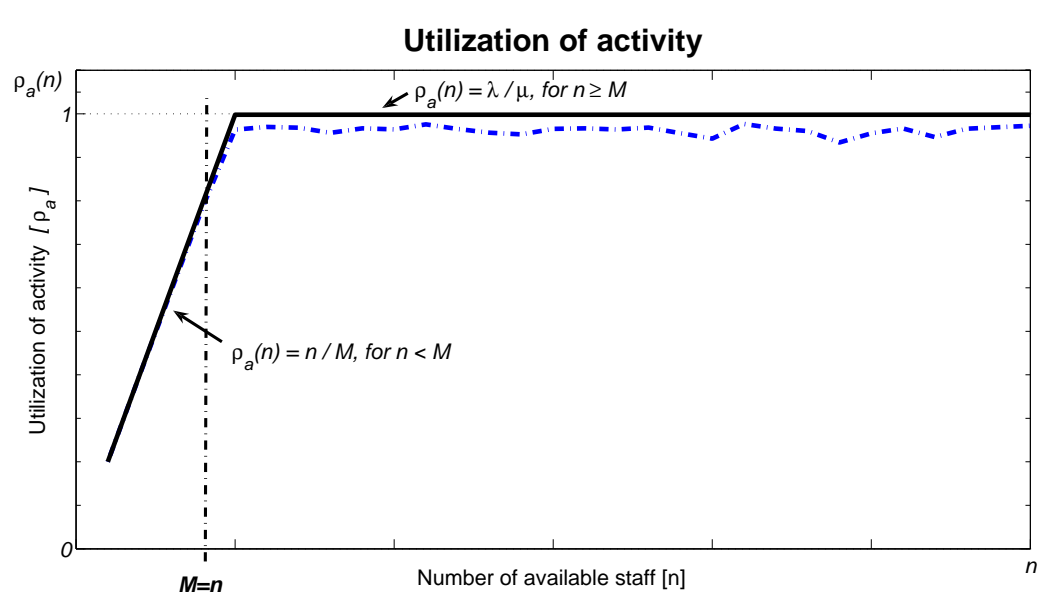

Fig. 5 The simulation result (dotted line) and the estimated calculated model (solid line) for the activity utilisation $\rho_{a}(n)$, which is a component of the overall utilisation $\rho(n)$.

Figure 5 illustrates the second component - the average utilization of activities $\rho_{a}(n)$ - on the blue dash-dotted line. In the case of understaffing, where fewer staff $\mathrm{n}$ are available than required to complete tasks $M$ ((i.e., where $n<M)$, some activities remain idle and waiting for staff, thus producing a linear trend. In such situations, utilization $\rho_{a}(n)$ is limited to the number of available staff, whereas in overstaffed conditions $(n \geq M)$, adequate or surplus staff mean the activity is permanently utilized, and is only limited to the ratio of the arrival rate $\lambda$ and the service time $\mu$. These observations can be summarized in the following equation which represents the utilization of an activity as:

$$
\rho_{a}(n, T)= \begin{cases}\frac{n}{M}, & \text { for } \frac{n}{M} \leq \frac{1}{\mu T} \text { and } n \leq M \\ \frac{1}{\mu T}, & \text { for } \frac{n}{M}>\frac{1}{\mu T} \text { and } n>M\end{cases}
$$

Equation 1 is valid for $\frac{1}{T} \leq \mu$. If utilization is not smaller than 1 , an unstable condition, which results in longer queues, occurs [11]. The term $\frac{n}{M} \leq \frac{\lambda}{\mu}$ limits the utilization of activity to the ratio of arrival rate to service time when there are more staff available than there are numbers of processes. The upper part of equation 1 describes the situation of understaffing; the lower part illustrates overstaffing (i.e. where the activities are at least adequately staffed) which depends on the arrival rate to service time ratio.

The utilization of staff $\rho_{s}(n)$ is the next component which is included in the overall utilization of the flexible workload environment $\rho(n)$. Figure 4 , which is obtained directly from the simulation, measures the utilization of staff who are busy with patients, i.e. those who are not waiting in the staff pool to be 


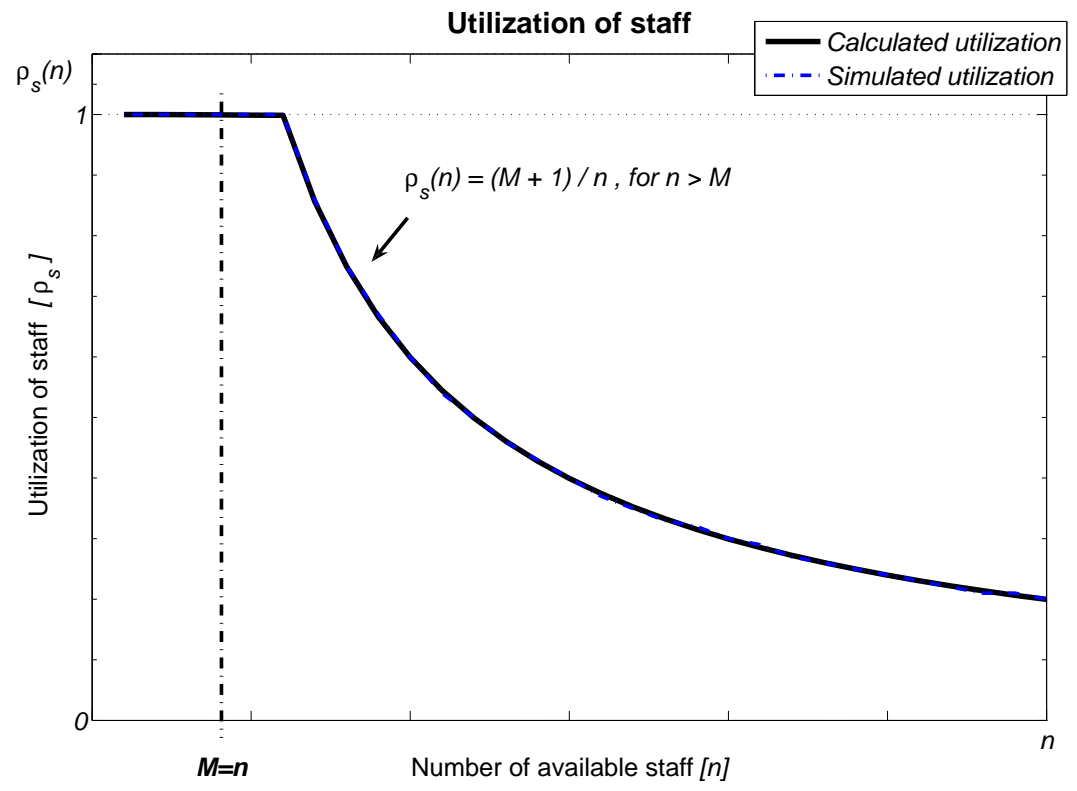

Fig. 6 The simulation result (dotted line) and the estimated calculated model (solid line) for staff utilization $\rho_{s}(n)$ for a specific arrival time slot $t$ depending on available staff $n$.

assigned to patients/tasks.

Figure 6 demonstrates that the utilization of staff is independent of patient arrival rate $\frac{1}{T}$ and service time $\mu$, and that the simulation result (blue dashdotted line) is equivalent to the estimated staff utilization as calculated in the simulation (solid line). It also shows that, in understaffing conditions (i.e., $n<M)$, staff utilization is equal to 1 - indicating that the staff are fully occupied by high workloads. When staff numbers are equal to the number of tasks $(n=M)$, their work is not interrupted and they do not need to alternate between tasks. When the process chain is overstaffed $(n \geq M+1)$, every additional staff member decreases the overall workload. This relationship can be summarized as:

$$
\rho_{s}(n)=\left\{\begin{array}{l}
1, \quad \text { for } n \leq M \\
\frac{M+1}{n}, \quad \text { for } n>M
\end{array}\right.
$$

Equation 2 is valid for all $\frac{1}{T}$ because staff utilization is obtained directly from the simulation model results. This equation is represented by the black line as displayed in Figure 5 which is equivalent to the trend observed. Having derived the mathematical expressions for both components (utilization of activity in equation 1 and utilization of staff in equation 2), the overall resulting 


\section{Resulting calculated overall utilization}

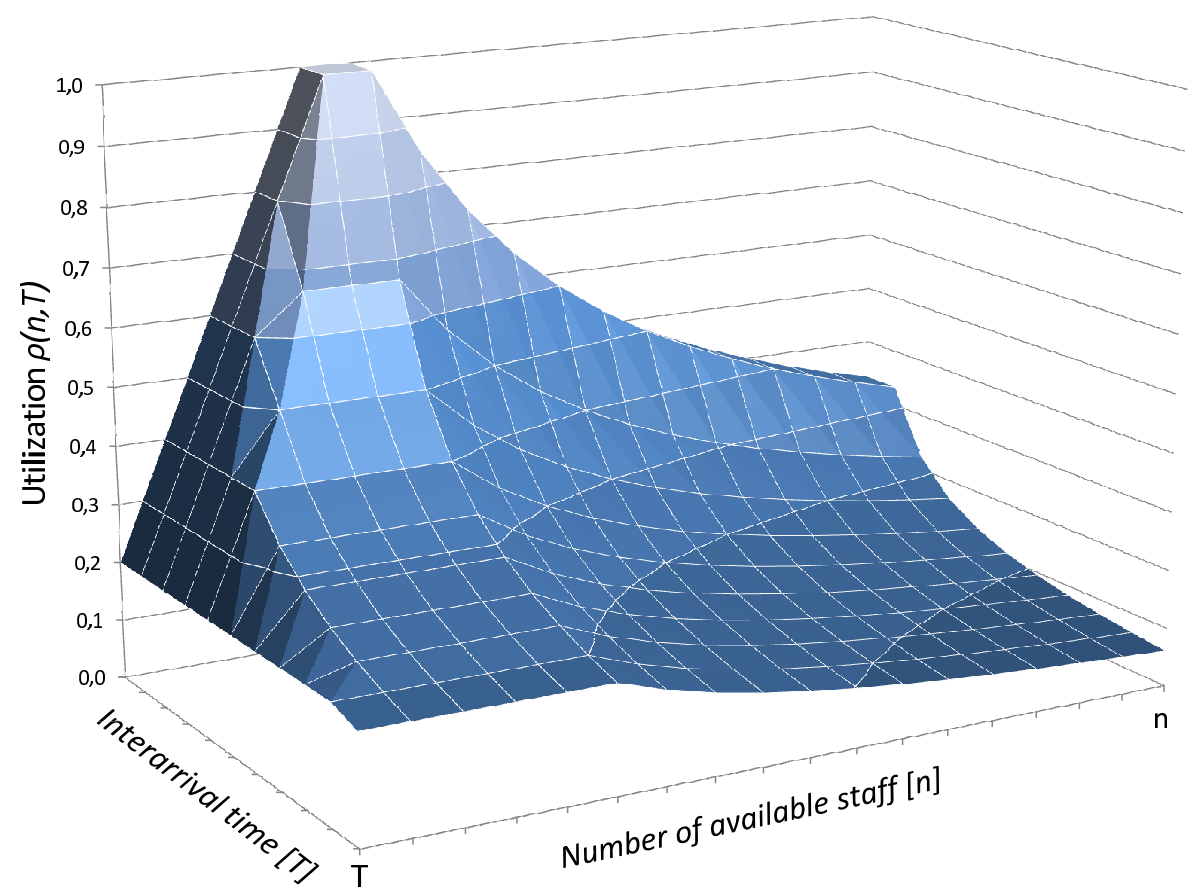

Fig. 7 Calculated overall utilization $\rho(n, T)$ according to the mathematical expression 3 given the utilization of staff $\rho_{s}(n)$ and activity $\rho_{a}(n, T)$.

utilization $\rho(n)$ can be calculated by multiplying the utilization of staff by the utilization of activities:

$$
\rho(n, T)=\rho_{s}(n) * \rho_{a}(n, T)
$$

Calculating the resulting utilization $\rho(n, T)$ taking inter-arrival times according to Equation 3 - into account, produces the graph shown in Figure 7. Comparing this to the outcome of the simulation results (Figure 3), it becomes obvious that the differences are due to the variability caused by random arrival rates and service rates triggered by the distribution. The variation however is relatively low because the results are calculated with an average of 1000 simulation runs. The utilization plot of the calculated model contains no 'fuzziness' because arrival and service times are constant. However, these can be integrated by incorporating the corresponding statistical distributions for arrival rates $\lambda$ and service times $\mu$ into equation 1 . The statistical comparison of the calculated spreadsheet model with the simulation results delivers a positive correlation of $99.91 \%$ for the whole model. 


\section{Interpretation of the Analytical Representation}

A pattern can thus be discerned - as described in Equation 3 - although minor variations exist due to the variance of patient arrival times and service time which is factored into equation 1 . The outcomes of equation 3 show similarities to the initial measurements (Figure 3), where the hyperbola axis are defined by the inter-arrival times $T$ and numbers of available staff $n$ (Figure 6). The plateau (Figure 7) marks the area where $\rho(n, T)=1$ under the condition that the inter-arrival time is set to $T \leq \mu$ and the number of available staff is less or equal to the number of tasks $(n<M)$. The slope on the left side of the plateau $(n<M)$ marks the area where the system tends to become unstable. The two graphs show that high utilization conditions are likely to occur when the available staff number $n$ is less or equal to the number of tasks $M$, and the arrival time is less than the service time. To avoid excessiv utilization of resources, the service time should either be less than the patient arrival time or surplus staff should be employed. As patient arrival times cannot normally be influenced by healthcare management, it is important to employ staff as the strategic variable.

\section{Analysis of the Analytical Representation}

Equation 3 represents the utilization of staff working in flexible workload environments such as an ED. It identifies the area of high utilization where $\rho(n, T)=1$ (as shown in Figure 7) which is a critical area where the system tends to become unstable, so that long queues are likely to develop. High utilization is also considered to be associated with an increased error rate which obviously should be prevented in critical environments such as healthcare [16, 19]. The example shown in Figure 8 illustrates how the proposed model is used to avoid severe understaffing conditions, and explains how the optimum number of staff is determined in order to achieve the desired utilization level while taking the uncertainty of the patient arrival rate $\lambda$ into account. Assuming the number of tasks within a process chain is $M=5$ and the inter-arrival time is set to $T=1.1 \mathrm{~h}$, real life observations show that the error rate in this model increases dramatically as the overall utilization level approaches $85 \%$. For this reason the function should not exceed this utilization level, which results in a staffing level of $n \geq 6$ in Figure 8 .

It is worth noting that the system can certainly be maintained with fewer numbers of staff than tasks available but this setting is limited to patient inter-arrival times being lower than service times $(T \leq \mu)$. Otherwise, queues build up between the processes. In our analytical representation we assume a constant flow of patients with queues (if any) being at least constant. Since this condition is impossible to control, optimizing staff numbers is required to avoid long and increasing waiting times due to the build up of queues. The 


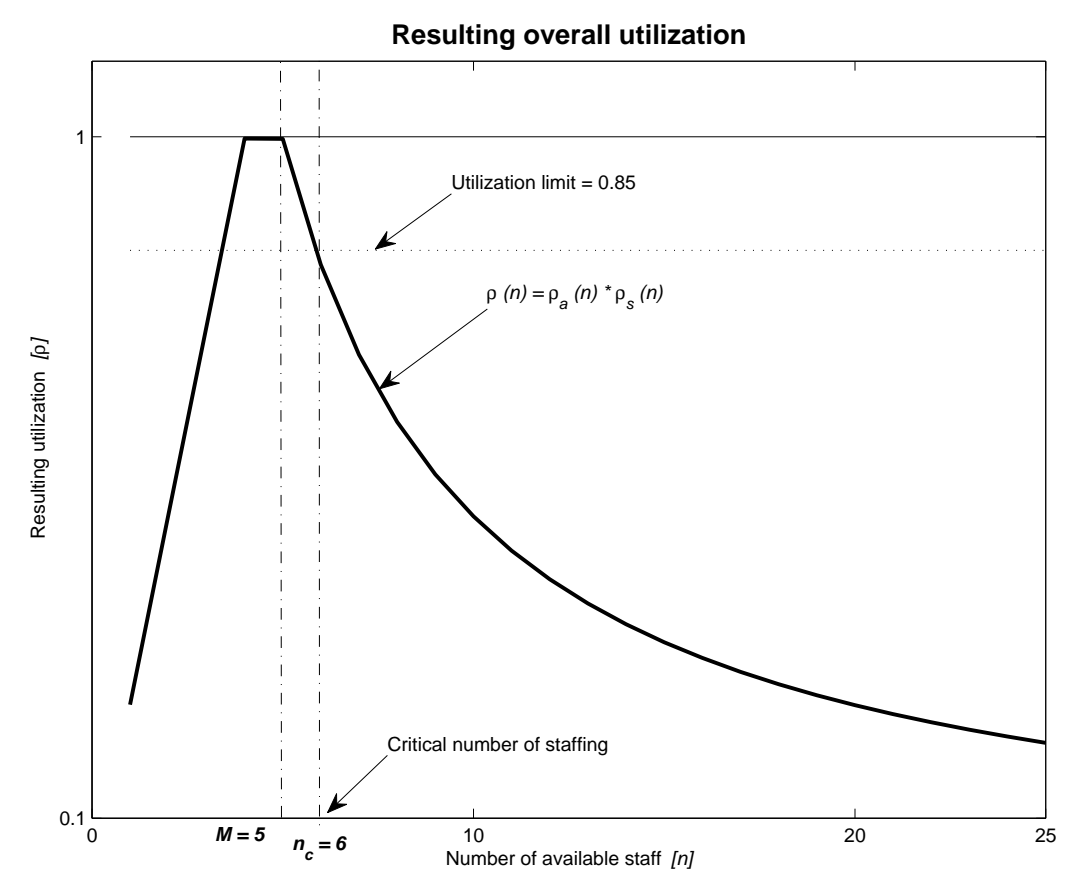

Fig. 8 An example of determining the critical number of staff $n_{c}$ for $M=5$ processes with a utilization limit of $\rho=85 \%$.

example in Figure 8 illustrates the guidance which the analytical representation can provide decision makers who attempt to deal with workloads that will lead to unstable queues if not adjusted properly. Patient arrival times are described as a Poisson distribution [8]. Healthcare managers can use the simulation model to examine the impact of changed inter-arrival times and other sources of variability to employ the right number of staff in the pool to achieve the desired overall staff utilization level. The analytical representation proposed here considers one type of skill set (i.e. nurses) - but, of course, in real life, a skill set mix (i.e. of doctors, consultants, administrative personnel) is involved in completing patients' care pathways [6].

Another important issue that should be taken into account is the fact that service times in process chains differ according to the nature of the tasks involved. In this process chain case (as shown in Figure 2), it is obvious that the service time of the longest task is likely to delay all the other processes, and becomes a bottleneck of which internal queues build up. Where the limitations of the analytical representation are too dominant for it to give useful guidance, decision makers will need to consult the original simulation model which considers multiple staff types and various process times. Although the analytical representation proposed here has these two limitations, it can still make the 
system's operating conditions more transparent for decision makers. This may result in a better understanding of the mechanisms operating in such flexible workload environments where high quality is crucial and expected. Furthermore, this analytical representation can help managers to make more accurate decisions on the staff numbers required to maintain service levels and avoid unstable conditions.

\section{Conclusion}

Identifying the correct resources of a process leads naturally to efficiency and quality in performance. Emergency Departments in hospitals often suffer from uncertain demands which require agile planning and reaction in order to ensure service quality. Cost implications are a source of pressure on managers of EDs with limited options forextra resources. On the other hand, an understaffed ED can result in seriously undesirable consequences such as high patient mortalityrates, stress and burnout among healthcare personnel, higher absenteeism rates among staff, lower work morale, and declining patient experiences. A stochastically uncertain queuing system such as in EDs may result in medical staff being excessively utilized and a significant increase in waiting times. Reports reveal higher mortality rates during high staff utilization periods and longer waiting times $[16,19]$.

A Discrete Event Simulation model was used to capture the dynamic nature of the process and understand the embedded variability within the ED in a leading university hospital in Dublin. This simulation model allowed managers to examine the dependency of staff utilization in the ED's three core units upon staff numbers and patient arrival times. An analytical representation was then integrated to address the staff utilization issue with a particular focus on nurses. The analytical representation developed reveals the burnout factor of nurses due to overutilization. Furthermore, it includes work with the simulation model to best schedule the staff according to a flexible workload. The key findings highlighted that the longest service time within the ED process chain should not exceed the patient arrival times and also staffing levels must not be lower than (or equal to) the numbers of discrete tasks within a process chain. If these two conditions are not met, the system will tend to be unstable, resulting in longer queues and high staff utilization (i.e. burnout), and hence a decline in service quality. These findings are expressed with a graph (Figure 7), generated with a spreadsheet calculation with consideration to patient arrival times and staff availability. The balance between resources mainly nurses in our example - and patient demand is crucial to maintaining satisfactory service quality and avoiding staff over-utilization. The proposed analytical representation helps decision makers and their feedback, after using the model, was highly positive. 


\section{References}

1. Abo-Hamad, W., Arisha, A.: A simulation-based framework to improve patient experience in an emergency department. European Journal of Operational Research 224(1)(1), 154-166 (2013)

2. Brailsford, S.: Overcoming the barriers to implementation of operations research simulation models in healthcare. Clinical and investigative medicine 28(6), 312-317 (2005). URL http://www.ncbi.nlm.nih.gov/pubmed/16450620

3. Friedman, L.W.: The Simulation Metamodel. Springer US (1996). DOI doi:10.1007/9781-4613-1299-4. ISBN: 978-1-4612-8556-4

4. Glasserman, P., Kou, S.G.: Analysis of an importance sampling estimator for tandem queues. ACM Trans. Model. Comput. Simul. 5, 22 - 42 (1995). DOI http://doi.acm.org/10.1145/203091.203093. URL http://doi.acm.org/10.1145/203091.203093

5. Harper, P., Shahani, A., Gallagher, J., Bowie, C.: Planning health services with explicit geographical considerations: a stochastic locationallocation approach. Omega 33(2), 141 - 152 (2005). DOI http://dx.doi.org/10.1016/j.omega.2004.03.011. URL http://www.sciencedirect.com/science/article/pii/S0305048304000593

6. Harper, P.R., Powell, N.H., Williams, J.E.: Modelling the size and skill-mix of hospital nursing teams. Journal of the Operational Research Society 61((2010)), 768-779 (2009)

7. Health Service Executive (HSE): Emergency department task force report. Tech. rep., Health Service Executive (HSE) (2007)

8. Isken, M.W., Ward, T.J., McKee Timothy, C.: Simulating outpatient obstetrical clinics. In: P.A. Farrington, H.B. Nemhard, D.T. Sturrock, G.W. Evans (eds.) Proceedings of the 31st conference on Winter simulation: Simulation - a bridge to the future - Volume 2, pp. 1557 - 1567. ACM, New York, NY, USA (1999)

9. Ismail, K., Thorwarth, M., Arisha, A.: Integrated decision support systems for improving emergency department performance in irish hospitals. International Journal of Operational Research 19(1), 40-59 (2014). DOI 10.1504/IJOR.2014.057843. URL http://inderscience.metapress.com/content/E55376000172685U

10. Jacobson, S.H., Hall, S.N., Swisher, J.R.: Patient flow: Reducing delay in healthcare delivery. Discrete-Event Simulation of Health Care Systems 91, 211-252 (2006)

11. Law, A.M., Kelton, W.D.: Simulation Modelling and Analysis, 3rd reviewed edition edn. McGraw-Hill Education, New York (2000)

12. Lowery, J.C.: Introduction to simulation in health care. In: D.T. Brunner, J.J. Swain, J.M. Charnes, D.J. Morrice (eds.) Proceedings of the 28th Conference on Winter Simulation, pp. 78-84. Coronado, California (1996)

13. Pidd, M.: Mixing other methods with simulation is no big deal. In: Simulation Conference (WSC), Proceedings of the 2012 Winter, pp. 1-7 (2012). DOI 10.1109/WSC.2012.6465280

14. Proudlove, N.C., Black, S., Fletcher, A.: OR and the challenge to improve the NHS: modelling for insight and improvement in in-patient flows. Journal of the Operational Research Society 58, 145-158 (2007)

15. Ragab, M., Rashwan, W., Abo-Hamad, W., Arisha, A.: Using modelling and simulation to improve elderly care in ireland: A case study. International Journal On Advances in Life Sciences 5(1 and 2), $89-102$ (2013)

16. Richardson, D.B.: Increase in patient mortality at 10 days associated with emergency department overcrowding. Medical Journal of Australia 184(5), 213-216 (2006)

17. Stahl, J.E., Rattner, D., Wiklund, R., Lester, J., Beinfeld, M., Gazelle, G.S.: Reorganizing the system of care surrounding laparoscopic surgery: A cost-effectiveness analysis using discrete-event simulation. Medical Decision Making 24(5), 461-471 (2004). DOI 10.1177/0272989X04268951. URL http://mdm.sagepub.com/content/24/5/461.abstract

18. Thorwarth, M.: A simulation-based decision support system to improve healthcare facilities performance elaborated on an Irish emergency department. Ph.D. thesis, Dublin Institute of Technology (2011)

19. Unruh, L., Joseph, L., Strickland, M.: Nurse absenteeism and workload: negative effect on restraint use, incident reports and mortality. Journal of Advanced Nursing 60(9), 673-681 (2007) 
20. Wierzbicki, A.P.: Modelling as a way of organising knowledge. European Journal of Operational Research 176(1), 610 - 635 (2007). DOI http://dx.doi.org/10.1016/j.ejor.2005.08.018. http://www.sciencedirect.com/science/article/pii/S0377221705007010

URL 